\title{
Vers une approche européenne de la formation des enseignants de langue?
}

Towards a European approach to training language teachers?

¿Hacia un enfoque europeo acerca de la formación de los profesores de lenguas?

Michael Kelly

\section{OpenEdition}

\section{Journals}

Édition électronique

URL : http://journals.openedition.org/ries/410

DOI : $10.4000 /$ ries.410

ISSN : 2261-4265

Éditeur

Centre international d'études pédagogiques

Édition imprimée

Date de publication : 1 avril 2008

Pagination : 45-54

ISBN : 978-2-85420-572-5

ISSN : 1254-4590

\section{Référence électronique}

Michael Kelly, « Vers une approche européenne de la formation des enseignants de lanque ? », Revue

internationale d'éducation de Sèvres [En ligne], 47 | avril 2008, mis en ligne le 27 juin 2011, consulté le 03 mai 2019. URL : http://journals.openedition.org/ries/410 ; DOI : 10.4000/ries.410 


\section{Vers une approche européenne de la formation des enseignants de langue?}

\section{Michael Kelly}

\section{Le CONTEXTE}

L'enseignement des langues prend une importance particulière au moment où les certitudes généreuses de l'Europe des Quinze sont confrontées aux exigences d'une Europe qui s'élargit et aux incertitudes d'un monde qui se rétrécit. Voies de communication et vecteurs d'identités, les langues sont en même temps un lieu et un enjeu des changements rapides qu'apporte la mondialisation. La formation des enseignants de langue prend donc une valeur stratégique dans la réponse européenne aux changements en cours.

Toute tentative de proposer des perspectives européennes dans ce domaine doit tenir compte du fait que l'enseignement à l'école ainsi que la formation des enseignants relèvent de la compétence nationale des pays membres. On constate également que, les systèmes nationaux se développant dans la diversité, il est nécessaire de respecter la spécificité de chacun afin d'apporter la meilleure réponse aux besoins particuliers de chaque situation nationale, voire régionale. Pourtant, les pays européens se trouvent de plus en plus sommés de renouveler leurs programmes pour faire face aux pressions qui les préoccupent tous.

La diversité est une valeur fondamentale de l'Europe qui rassemble des systèmes multiples. Elle est aussi une riche source d'innovations. On oublie trop facilement que le progrès surgit bien souvent de solutions inédites à des problèmes communs, et que la solution unique peut facilement empêcher des solutions mieux adaptées. Évidemment, c'est un prix à payer en termes de complexité voire d'incohérences. D'autant qu'originalité et efficacité ne vont pas toujours de pair. On cherchera à relativiser ces inconvénients à travers les échanges d'idées et en favorisant les convergences volontaires. Comment, donc, favoriser les coopérations entre les pays européens pour mieux conjuguer leurs efforts dans ce domaine?

C'est de cette question et de ces difficultés qu'est né le projet de profil pour la formation des enseignants de langue (Kelly et Grenfell 2004). On pourrait opportunément citer Charles Péguy, pour qui «tout commence en 
mystique et finit en politique», pour saisir l'itinéraire qu'il faudra suivre. Cela ne signifie pas que ceux qui produisent des idées n'ont pas à se salir les mains dans la cuisine de la politique et de la pratique. Il s'agit plutôt de proposer des moyens concrets qui permettent d'élaborer une politique de l'enseignement susceptible de mettre en œuvre la «mystique» des aspirations européennes.

Face à la très grande diversité de pratiques et de systèmes, il fallait d'abord trouver un vocabulaire partagé qui permette de mener un dialogue efficace. Il fallait ensuite esquisser de grandes lignes de réflexion pour nourrir le travail de renouvellement auquel se consacrent les enseignants et les décideurs des pays européens. Nous montrerons comment le projet de profil s'est efforcé d'offrir une trousse d'outils en termes de principes généraux et de traduction en pratique.

\section{UNE PROBLÉMATIQUe MULTIPLE}

$\mathrm{Au}$ départ la question posée peut sembler assez simple : comment améliorer les capacités linguistiques des Européens? La nécessité de développer les compétences en langues est reconnue depuis longtemps et fait partie de la stratégie de Lisbonne de 2000, où elles figurent comme l'une des cinq compétences de base pour favoriser la croissance économique et l'emploi. Déjà, la Commission avait adopté l'objectif, confirmé par le conseil des ministres, que tout citoyen doit pouvoir parler deux langues outre sa langue maternelle. La motivation économique est certes la plus importante, mais des motivations politiques et sociales se dessinent également dans les documents officiels qui s'orientent plutôt vers l'inclusion sociale au sein de chaque pays, et sur les relations culturelles entre les peuples. Plus récemment, ces motivations sont présentées sous l'angle de la diversité, principe fondamental du projet de construction européenne, et qui ne manque pas d'embrasser d'autres aspirations, et notamment le besoin de faire valoir une identité nationale, régionale ou ethnique.

Tout indique donc la nécessité d'améliorer les capacités linguistiques. Pour y parvenir, le rôle de l'enseignement est incontournable. S'agit-il cependant de renforcer l'enseignement tel qu'il existe déjà? Il est sans doute souhaitable de faire plus, mais ne faudrait-il pas en même temps faire mieux? On peut difficilement le nier. Et pourtant, il n'est pas facile de créer des cursus et des pédagogies mieux adaptés à des motivations de plus en plus complexes. Le choix de langues à apprendre n'est plus une évidence. Tout comme le choix des méthodes pédagogiques. Dans ces conditions, comment envisager une formation initiale capable de fournir les outils de base nécessaires pour faire face à ce contexte dynamique? Et quelle formation continue pourrait répondre aux besoins en évolution que rencontreront les enseignants au long de leur carrière? C'est à ces questions que nous avons essayé d'apporter des éléments 
de réponse dans l'étude que nous avons menée pour proposer un profil de la formation des enseignants de langue. Mais il a fallu partir des réalités actuelles, qui s'inscrivent sous le signe de la diversité.

\section{DIVERSITÉ DES FORMATIONS}

La première partie de notre étude était conçue comme une typologie des formations. En effet, à travers une trentaine de pays européens, on constate une diversité très riche (Grenfell, Kelly et Jones 2002). On retiendra quelques exemples en ce qui concerne l'organisation pratique. La formation des instituteurs au niveau de l'école primaire est assez différente de celle qui est offerte pour le secondaire, lui-même souvent divisé en deux phases. Des formations sont proposées pour au moins trente langues. Le lieu de formation est très variable, le plus souvent mais pas toujours dans une institution universitaire, où il est en général partagé entre les instituts de langues modernes et les instituts pédagogiques. Les écoles et lycées jouent un rôle très important dans la formation. Les conditions d'étude varient entre salaires garantis, bourses et droits d'inscription. Les diplômes sont proposés à plusieurs niveaux, surtout au niveau de la licence et en deuxième cycle, et souvent contrôlés par une instance gouvernementale comme un ministère ou une agence spécialisée. Ils aboutissent à des statuts d'emploi assez différents qui vont du fonctionnariat à poste assuré jusqu'au marché du travail général. Bien sûr, la reconnaissance des diplômes d'un pays à l'autre reste assez limitée.

Le contenu des formations fait montre de la même diversité. Laissant de côté les études plus générales (histoire, philosophie, psychologie, sociologie de l'éducation), pour ne considérer que l'enseignement des langues: on constate que l'apprentissage d'une langue elle-même y occupe une place variable jusqu'à en être totalement absent (la compétence étant présumée acquise), comme c'est le cas en Angleterre par exemple. Il en va de même pour la grammaire et la linguistique appliquée. La formation porte souvent sur une seule langue, mais de plus en plus souvent sur deux langues ou sur deux sujets où la langue est combinée à une autre matière (souvent l'histoire ou la géographie). Certains pays insistent pour que la formation se poursuive dans la langue à enseigner, suivant par exemple la méthode EMILE/CLIL, qui prend de plus en plus d'ampleur. D'autres, comme la plupart des universités britanniques, s'en tiennent à une langue d'instruction commune à toutes les matières. On pourrait facilement multiplier les exemples de divergences d'un pays à l'autre, et souvent d'une région à l'autre dans un même pays.

C'est en dressant un recensement des différences que nous avons appris à formuler les ressemblances. Et nous nous sommes rendu compte que les éléments qui étaient articulés de façon explicite dans un pays étaient souvent implicites dans un autre, alors qu'on aurait pu croire qu'ils étaient absents. C'est ce constat qui est 
à la base de notre projet de créer un document de référence capable d'offrir un vocabulaire commun. C'était aussi la possibilité de recenser les bonnes pratiques méconnues qui mériteraient de connaître une diffusion plus large.

\section{VERS UN VOCABULAIRE COMMUN}

Le premier pas vers l'établissement d'un profil de la formation des enseignants de langue est le recensement des éléments divers que l'on peut trouver dans les programmes de formation. Nous y avons procédé à partir de l'étude de trente-trois pays. Dès le départ, ce fut un travail d'inventaire qui visait la gamme quasi complète des composants possibles.

Nous avons rassemblé les idées-clés par un processus intensif de consultations : d'une part en faisant appel à une dizaine d'experts de sept pays qui ont participé à une série d'entretiens autour des grands thèmes; d'autre part en confiant à l'équipe de recherche l'étude d'une douzaine de cas susceptibles de faire apparaître des conceptions et des pratiques efficaces dans des contextes bien différents.

Il est apparu bien rapidement qu'aucun cas particulier ne rassemblait tous les éléments possibles du curriculum. La diversité linguistique implique aussi que certains concepts n'existent pas dans certaines langues, ou sont compris dans des concepts plus larges, et qu'un terme apparemment identique dans deux langues peut très bien désigner des réalités différentes.

On s'aperçoit, par exemple, que la notion de «stage pratique» fait l'objet d'appellations diverses, dont certaines sont retenues dans la troisième recommandation du Profil. En français, nous proposons «un cadre explicite pour le stage pratique d'enseignement»; en allemand, cela devient «expliziter Rahmen für die Unterrichtspraxis (Stage/Praktikum)», et en anglais, «an explicit framework for teaching practice (stage/practicum)», (Kelly et Grenfell 2004). On constate que pour les étudiants en formation, l'intervention dans les classes peut prendre plusieurs formes et qu'un «stage» est entouré d'attentes différentes du «Praktikum» ou de la «teaching practice». La réalité vécue varie de quelques jours, pendant lesquels l'étudiant observe le travail d'un enseignant professionnel, à quelques mois de cours à assurer seul en classe, quitte à recevoir des visites d'observateurs et d'inspecteurs.

En fait, la présentation plurilingue de cette recommandation a l'avantage de rappeler que la pratique particulière d'un pays relève d'un choix, et pourra mener à une réflexion sur la raison d'être de cette pratique de l'enseignement. Qui plus est, en regardant ces différences, il devient encore plus évident qu'il faut rendre explicite les modalités et les finalités du stage. On ne peut faire l'économie de cette sorte de réflexion si l'on veut parvenir à un vocabulaire commun qui permettra aux formateurs et aux décideurs européens de se parler et de se comprendre. Pour réussir, leur dialogue tiendra nécessairement compte de ces complexités. 


\section{CONCEVOIR UN CADRE DE RÉFÉRENCE}

La réflexion sur le sens social et la résonance sémantique des concepts permet donc de construire un vocabulaire. Si cela prend son sens dans le dialogue, le dialogue lui-même prend son sens dans les tâches qu'il permet d'accomplir. Dans le cas présent, la tâche, rappelons-le, est de favoriser les convergences et les coopérations entre les pays pour mieux conjuguer leurs efforts dans le domaine de la formation des enseignants de langue. C'est dans cette perspective que nous avons essayé de trier les concepts pour construire un profil des éléments jugés les plus importants.

Pour ce travail de sélection, nous avons fait appel à vingt-quatre formateurs actifs, dans une vingtaine de pays, qui ont accepté de participer à une enquête DELPHI. Cette méthode repose sur une série de questionnaires conçus de façon à établir les perspectives communes du groupe. Dans un premier temps, nous avons demandé aux participants de livrer leurs réflexions sur la structure et le contenu des programmes, les voies d'amélioration et les perspectives de coopération européenne. Leurs réflexions ont été résumées dans une longue liste de propositions, auxquelles nous avons demandé dans un deuxième temps aux participants de réagir. Dans un troisième temps, ils ont indiqué l'importance qu'ils attachaient aux éléments les plus commentés. Par la suite, les résultats ont été discutés par l'ensemble des experts.

Le Profil dressé à la fin de ce processus comporte quarante éléments auxquels les formateurs sont invités à réfléchir (voir l'annexe en fin d'article). On distinguera quatre types d'éléments :

- la structure et l'organisation des différentes parties constitutives de la formation des enseignants de langues;

- les savoirs et les compétences que la formation doit assurer par rapport à l'enseignement et à l'apprentissage des langues;

- les stratégies et le savoir-faire que les enseignants formés doivent être capables d'apporter dans différents contextes d'enseignement et d'apprentissage ;

- les valeurs que les futurs enseignants de langues doivent apprendre à promouvoir dans et au travers de leur enseignement.

Ces quatre catégories doivent bien sûr être considérées d'un point de vue holistique, puisque la formation des enseignants est une activité multidisciplinaire à plusieurs facettes, où les éléments s'interpénètrent. Dans la liste élargie, certains éléments se retrouveront plutôt dans la formation continue (par exemple l'évaluation des programmes) alors que d'autres s'appliquent évidemment à la formation initiale (par exemple le stage).

Pour certains, beaucoup d'éléments sembleront aller de soi. Mais nous avons constaté qu'il y en a peu qui ne fassent pas de débat. Le stage lui-même, sous quelque forme qu'il soit compris, est loin d'être une évidence. Certains 
pays s'en tiennent à l'aspect théorique de la formation ou bien limitent à certaines catégories de formation l'obligation d'incorporer un élément pratique. L'importance du stage est pourtant reconnue à l'unanimité par tous ceux qui ont contribué au projet de recherche, si bien qu'ils tombent d'accord sur la nécessité d'appeler, dans la toute première recommandation, à «un programme d'études intégrant la formation théorique et l'expérience pratique de l'enseignement». C'est un appel à double tranchant, puisqu'il vise aussi les pays (peu nombreux, il est vrai) où l'enseignant en primaire ou en secondaire ne reçoit aucune formation théorique avant de se lancer dans cette carrière. Il est vrai que la formation des enseignants de langue dans les universités reste extrêmement aléatoire - mais c'est sans doute un autre débat.

\section{LA BOÎTE À OUTILS}

Chaque titre du Profil a été longuement discuté, et s'associe dans le rapport intégral non seulement à une explication plus élaborée, mais aussi à des stratégies pour sa mise en œuvre et son application, et à quelques exemples d'actualité puisés dans les études de cas. Chaque titre correspond ainsi à des pratiques réelles dont on peut consulter trois ou quatre exemples dans des pays européens. Ce qui est proposé est donc une boîte d'outils, disponibles pour qui veut repenser la formation offerte aux enseignants de langue, ou qui veut simplement explorer des possibilités supplémentaires. Son utilité a été reconnue par la Commission européenne, qui le recommande aux pays membres dans sa communication sur le multilinguisme (Commission européenne 2005 : 7). Étant donné la diversité des systèmes, il n'est pas question de chercher l'uniformité, ni a fortiori d'imposer des règles universelles. On propose plutôt une série d'invitations à la réflexion.

Manier ces outils exige à la fois une certaine ouverture d'esprit et un brin d'imagination. Ils proviennent d'un processus d'élaboration qui a favorisé des principes larges et intelligibles plutôt que des instructions détaillées. Il s'agira de chercher à voir comment se présente le problème dans le contexte national ou régional particulier, alors qu'il est posé en termes généraux dans le profil. Il faut ensuite imaginer comment la solution proposée en termes généraux pourrait s'articuler dans ces circonstances spécifiques. Le cas du tutorat/monitoring en est un bon exemple.

Le problème général auquel cela renvoie surgit quand les enseignants en formation se retrouvent dans l'école ou le lycée où ils suivent leur stage pratique. Ils peuvent se sentir déboussolés dans ces premiers contacts avec la pratique de l'enseignement. Ou, tout au moins, ne pas profiter suffisamment de cette expérience. Dans plusieurs pays, on considère qu'une façon efficace de réduire ce problème est l'intervention d'un tuteur/mentor, qui pourra faciliter leur insertion dans la pratique de l'enseignement. Or, comme nous l'avons vu, le contact de l'étudiant/stagiaire avec l'école varie énormément à travers 
l'Europe, ainsi que les rapports entre les formateurs et les enseignants en établissement. Le soutien que pourrait offrir le tuteur/mentor varie également. C'est peut-être pour cette raison que les concepts de «mentor» et «tuteur» ne sont pas tout à fait équivalents. Pour cette raison, les traductions allemandes et roumaines ont choisi de retenir le terme "monitoring», pour signaler qu'il s'agit d'un rôle qui se distingue des fonctions d'enseignant. Là où les stagiaires passent une longue période en stage, ils ont peut-être davantage besoin d'un accompagnement de ce genre. Dans cette situation, il est de coutume qu'un enseignant en établissement soit désigné pour accompagner de près un stagiaire. Cela fait souvent partie de ses responsabilités formelles ou peut valoir une rémunération supplémentaire. Par contre, là où les stages sont courts, l'accompagnement est souvent assez léger, n'est pas toujours formalisé, et est assuré dans certains cas par le formateur universitaire.

La mise en pratique d'un système de mentor/tuteur dépend des conditions dans lesquelles les stages pratiques prennent place. L'essentiel est de soutenir les stagiaires tout au long de leur stage, en accompagnant la transition entre le stade de stagiaire et celui d'enseignant diplômé. L'avantage du mentor situé dans l'établissement, c'est aussi d'assurer une meilleure coopération entre l'université et l'école.

Il semble maintenant acquis que la formation des enseignants de langues concerne toute l'Europe. D'abord, cette formation joue un rôle stratégique dans le projet européen. Ensuite, les formateurs ont un rôle important dans les échanges linguistiques. Et en dernier lieu, du point de vue pratique, les formateurs européens constatent qu'ils ont beaucoup à apprendre les uns des autres. C'est à ce rendez-vous des intérêts partagés que notre étude apporte à la fois des perspectives théoriques et des outils pratiques. Nous espérons que cet apport permettra d'adapter les profils des enseignants, à travers leur formation, aux exigences de leurs missions dans des systèmes éducatifs dont les objectifs, en matière d'enseignement des langues, sont en pleine transformation. Le travail de renouvellement s'inscrit sous le signe de la diversité, nécessaire à l'innovation comme au respect des identités. Mais il doit aussi s'inscrire sous le signe de la coopération, qui sera de plus en plus nécessaire à l'efficacité comme à la découverte de l'autre. Les liens de solidarité ainsi renforcés permettront aux enseignants, aux formateurs et aux décideurs de donner à l'Europe des capacités linguistiques accrues.

Ces avancées ne sont pas assurées. La coopération n'est jamais acquise, mais toujours à renouveler. Beaucoup de pressions s'exercent contre elle. La question des moyens financiers n'est pas la plus difficile à résoudre. Il faut dégager le temps nécessaire dans des emplois du temps déjà chargés, et 
renforcer la motivation des enseignants et de leurs formateurs à s'engager dans une voie où les joies sont assorties de fardeaux administratifs. Mais dans le fond, les obstacles se cachent dans les esprits et les cultures. Enseigner une langue, c'est en quelque sorte devenir l'ambassadeur d'une culture - ou des cultures - portée(s) par elle, avec les obligations et les fiertés qui vont de pair. Comment encourager les enseignants d'une langue à devenir des enseignants de langue dans un sens plus général? Là, nous touchons à des sensibilités nationales où les affinités électives sont parfois plus fortes que celles de l'état civil. Pourrons-nous faire en sorte que ces solidarités ne deviennent pas des exclusivités? L’avenir de la diversité européenne pourrait en dépendre.

\section{BibliogRAPHIE}

Commission européenne (2005): Communication de la Commission au conseil, au parlement, au comité économique et social européen et au comité des régions. «Un nouveau cadre stratégique pour le multilinguisme». COM (2005) 596 final. Place Published. http://europa.eu/languages/servlets/Doc? id=914 (consulté le 13 janvier 2008).

GRENFELL Michael, KELLY Michael et JONES Diana (2002): The Training of Teachers of a Foreign Language : Developments in Europe. Place Published. http://ec.europa.eu/education/policies/lang/doc/executive_summary_full_en.pdf (consulté le 3 janvier 2008).

KELLY Michael et GRENFELL Michael (2004): Europäisches Profil für die Aus- und Weiterbildung von Sprachenlehrkräften : ein Referenzrahmen. Place Published. http: //ec.europa.eu/education/policies/lang/doc/profile_de.pdf (consulté le 2 janvier 2008).

(2004): European Profile for Language Teacher Education - A Frame of Reference. Place Published. http://ec.europa.eu/education/policies/lang/doc/profile_ en.pdf (consulté le 2 janvier 2008).

(2004) : Profil européen pour la formation des enseignants de langues étrangères - un cadre de référence. Place Published. http://ec.europa.eu/education/policies/ lang/doc/profilebroch_fr.pdf (consulté le 2 janvier 2008).

PÉGUY Charles (1933): Notre jeunesse. Paris : Gallimard. 


\section{Résumé du Profil}

\section{Structure}

1. Un programme d'études intégrant la formation théorique et l'expérience pratique de l'enseignement (le stage).

2. L'agencement flexible et modulaire de la formation initiale et continue.

3. Un cadre explicite pour le stage pratique d'enseignement.

4. Le travail avec un tuteur ainsi que dans l'appréciation de la valeur du tutorat.

5. L'expérience d'un environnement interculturel et multiculturel.

6. Le développement de liens avec des partenaires à l'étranger (séjours, échanges et liens TIC).

7. Une période de travail ou d'étude dans un ou plusieurs pays dont la langue étrangère est celle que le stagiaire enseigne.

8. L'occasion d'observer l'enseignement et d'y participer dans plus d'un pays.

9. Un cadre d'évaluation européen pour les programmes de formation initiale et continue des enseignants, permettant ainsi l'accréditation et la mobilité.

10. Un programme de perfectionnement méthodologique dans le cadre de la formation continue.

11. La formation continue des formateurs d'enseignants.

12. Une formation au tutorat pour les tuteurs en situation scolaire.

13. Des liens étroits entre les stagiaires formés à enseigner des langues différentes.

\section{Savoirs et compétences}

14. Une formation aux méthodologies d'enseignement des langues et aux techniques et activités de classe les plus avancées.

15. Une formation au développement d'une approche critique et de questionnement de l'enseignement et de l'apprentissage.

16. Une formation initiale à l'enseignement incluant un module sur les compétences linguistiques permettant l'évaluation de la compétence linguistique du stagiaire.

17. Une formation aux technologies de l'information et de la communication pour leur emploi pédagogique dans la salle de classe.

18. Une formation aux technologies de l'information et de la communication dans le domaine de la planification personnelle, de l'organisation et de la découverte de ressources.

19. Une formation à l'administration des procédures d'évaluation diverses et aux façons de suivre le progrès des apprenants.

20. Une formation à l'évaluation des programmes adoptés à l'échelon national ou régional en fonction de leurs buts, objectifs et résultats.

21. Une formation à la théorie et à la pratique de l'évaluation interne et externe des programmes. 


\section{Stratégies et savoir-faire}

22. Une formation aux façons d'adapter la manière d'enseigner au contexte éducatif et aux besoins individuels des apprenants.

23. Une formation à l'évaluation critique, au développement et à l'utilisation des supports et ressources pédagogiques.

24. Une formation aux méthodes d'apprendre à apprendre.

25. Une formation au développement de la pratique réflexive et de l'auto-évaluation.

26. Une formation au développement de stratégies spécifiques à l'apprentissage autonome des langues.

27. Une formation aux méthodes visant à entretenir et améliorer en continu ses propres compétences langagières.

28. Une formation à l'application pratique des parcours et programmes scolaires.

29. Une formation aux techniques d'observation et de correction de pairs.

30. Une formation au développement des relations avec des institutions d'éducation dans des pays choisis.

31. Une formation à la recherche-action.

32. Une formation à l'intégration de la recherche à l'enseignement.

33. Une formation à l'Enseignement d'une Matière par l'Intermédiaire d'une Langue Étrangère (EMILE).

34. Une formation à l'utilisation du Portfolio européen des langues pour l'auto-évaluation.

\section{Valeurs}

35. Une formation aux valeurs sociales et culturelles.

36. Une formation à la diversité des langues et des cultures.

37. Une formation à l'importance de l'enseignement et de l'apprentissage concernant les langues et cultures étrangères.

38. Une formation à l'enseignement de la citoyenneté européenne.

39. Une formation au travail d'équipe, à la coopération et au travail en réseau, à l'intérieur et à l'extérieur du contexte immédiat de l'école.

40. Une formation à l'importance de l'apprentissage tout au long de la vie. 